\title{
Complete Instability of Differential Inclusions using Lyapunov Methods
}

\author{
Philipp Braun $^{1,2}$, Lars Grüne ${ }^{2}$, Christopher M. Kellett ${ }^{1}$
}

\begin{abstract}
Lyapunov functions and control Lyaupunov functions are a well established tool in the analysis of stability properties of dynamical systems as well as in the design of stabilizing feedback controllers. In order to address problems such as stabilization in the presence of unsafe sets of states or obstacle avoidance, one potential approach involves rendering such obstacles unstable by feedback. To this end we introduce (nonsmooth) Chetaev and control Chetaev functions and demonstrate their sufficiency for complete instability properties of dynamical systems. While a "time-reversal" approach is frequently used to study instability in reverse time of an asymptotically stable point in forward time, we demonstrate via an example that such an approach cannot be used to generate Chetaev functions from nonsmooth Lyapunov functions via a simple change of sign in the time argument.
\end{abstract}

\section{INTRODUCTION}

Lyapunov functions (LFs), originating in [16], are a well established tool to analyze and characterize stability and instability properties of equilibria of ordinary differential equations (ODEs). For dynamical systems with inputs and for differential inclusions, the concept of LFs has been extended to control LFs (CLFs) [2], [20], and it was shown that the existence of a nonsmooth Lipschitz continuous CLF is equivalent to the stabilizability of a target set of a dynamical system. The necessary and sufficient conditions for the existence of CLFs have subsequently been derived in [20], [18] and [12].

While the theory for LFs and CLFs is quite mature, very little has been done towards deriving necessary and sufficient conditions for the existence of Lyapunov-type functions characterizing instability properties of equilibria for dynamical systems and differential inclusions. Indeed, the most general results describing instability of ordinary differential equations using Lyapunov-like functions dates back to Chetaev [6], with converse results derived in [13], [22] (see [11]).

However, these results are not applicable to the problems of "robust instability" of differential inclusions or "destabilizability" of control systems. The ability to (locally) destabilize a point or set is important in safety critical applications as well as obstacle and collision avoidance, where not only must a target set be stabilized, but additionally, unsafe states or obstacles need to be avoided. One way to accomplish

\footnotetext{
* The authors are supported by the Australian Research Council (Grant number: ARC-DP160102138).

1 P. Braun and C. M. Kellett are with the School of Electrical Engineering and Computing at the University of Newcastle, Callaghan, New South Wales 2308, Australia, \{chris.kellett, philipp.braun\}@newcastle.edu.au.

${ }^{2} \mathrm{P}$. Braun and L. Grüne are with the Mathematical Institute, Universität Bayreuth, 95440 Bayreuth, Germany, \{philipp.braun, lars.gruene\}@uni-bayreuth.de.
}

this is to render such unsafe states locally unstable (see for example [23] or [1]). A rigorous understanding of instability properties of dynamical systems is thus a necessary first step in the design of uniting controllers which stabilize a target set and destabilize unsafe states (cf. [19], [1] [4], and [5]).

Inspired by the use of CLFs for stabilizing feedback design, control barrier functions were introduced in [23] as a tool to avoid unsafe states. However, control barrier functions do not exactly mirror the definitions of CLFs characterizing stabilizability. In order to develop a more direct analogue of CLFs, we introduce Chetaev functions (CFs) and control Chetaev functions (CCFs), acknowledging the work of Chetaev [6], to characterize instability and destabilizability properties of differential inclusions, mirroring existing results on nonsmooth (control) LFs. Note that smooth CCFs were proposed for control-affine systems in [9] though no proofs were provided.

The paper is organized as follows: In Section II, the mathematical setting is introduced, i.e., differential inclusions describing dynamical systems are defined, and the Dini derivative, used for nonsmooth CLFs and CCFs, is discussed. Section III recaps known Lyapunov results on stability and instability of dynamical systems characterized through LFs and CLFs. The main results of the paper are discussed in Section IV. Here, CFs and CCFs are introduced and sufficient conditions for complete instability and destabilizability are provided. In Section V we investigate connections between stabilizability in forward time and destabilizability in backward time as well as corresponding implications on the existence on CLFs and CCFs. The paper concludes in Section VI.

The following notations are used throughout the paper. The norm of a vector $x \in \mathbb{R}^{n}$ is denoted by $|x|$. For $x \in \mathbb{R}^{n}$ we use $B_{\varepsilon}(x)=\left\{y \in \mathbb{R}^{n}|| x-y \mid<\varepsilon\right\}$ to denote the open ball of radius $\varepsilon>0$, centered around $x$. For two sets $\mathcal{A}, \mathcal{B} \subset \mathbb{R}^{n}$, $\mathcal{A}+\mathcal{B}$ denotes the Minkowski sum, i.e., $\mathcal{A}+\mathcal{B}=\{a+b \mid a \in$ $\mathcal{A}, b \in \mathcal{B}\}$. The closure of a set $\mathcal{A} \subset \mathbb{R}^{n}$ is denoted by $\overline{\mathcal{A}}$ and $\overline{\operatorname{conv}}(\mathcal{A})$ denotes the closure of its convex hull.

The stability results will be based on so-called comparison functions where we refer to [10] for details and comprehensive results. A continuous function $\rho: \mathbb{R}_{\geq 0} \rightarrow \mathbb{R}_{\geq 0}$ is said to be of class $\mathcal{P}(\rho \in \mathcal{P})$ if $\rho(0)=0$, and $\rho(s)>0$ for all $s>0$. A function $\alpha \in \mathcal{P}$ is said to be of class $\mathcal{K}(\alpha \in \mathcal{K})$ if it is strictly increasing. A function $\alpha \in \mathcal{K}$ is said to be of class $\mathcal{K}_{\infty}\left(\alpha \in \mathcal{K}_{\infty}\right)$ if $\lim _{s \rightarrow \infty} \alpha(s)=\infty$. A continuous function $\sigma: \mathbb{R}_{\geq 0} \rightarrow \mathbb{R}_{\geq 0}$ is said to be of class $\mathcal{L}(\sigma \in \mathcal{L})$, if it strictly decreasing, and $\lim _{s \rightarrow \infty} \sigma(s)=0$. A continuous function $\beta: \mathbb{R}_{>0}^{2} \rightarrow \mathbb{R}_{\geq 0}$ is said to be of class $\mathcal{K} \mathcal{L}(\beta \in \mathcal{K} \mathcal{L})$ if $\beta(\cdot, s) \in \mathcal{K}_{\infty}$ for all $s \in \mathbb{R}_{\geq 0}$ and $\beta(s, \cdot) \in \mathcal{L}$ for all 
$s \in \mathbb{R}_{\geq 0}$.

\section{Mathematical Setting}

In this paper are we interested in stability and instability properties of equilibria for differential inclusions characterized via Lyapunov arguments. Since in this context smooth control Lyapunov functions (CLFs) are not sufficient to describe stability properties, we will use nonsmooth CLFs in the Dini sense [20], which we will discuss in this section.

\section{A. Differential inclusions}

In this paper we consider dynamical systems described through a differential inclusion

$$
\dot{x} \in F(x), \quad x_{0} \in \mathbb{R}^{n},
$$

for a set-valued map $F: \mathbb{R}^{n} \rightrightarrows \mathbb{R}^{n}$, and an initial value $x(0)=x_{0} \in \mathbb{R}^{n}$. We are interested in stability properties of the origin and assume that without loss of generality $0 \in$ $F(0)$ holds. To guarantee existence of solutions of (1) we will make the following assumption on $F$ throughout the paper.

Assumption 2.1: Consider the set-valued map $F: \mathbb{R}^{n} \rightrightarrows$ $\mathbb{R}^{n}$ with $0 \in F(0)$. Additionally, we impose the following conditions on $F$ :

(i) $F$ has nonempty, compact, and convex values on $\mathbb{R}^{n}$, and it is upper semicontinuous.

(ii) For each $r>0$ there exists $M>0$ such that $|x|<r$ implies $\sup _{w \in F(x)}|w| \leq M$.

(iii) $F$ is Lipschitz continuous on $\mathbb{R}^{n} \backslash\{0\}$.

The compact set-valued map $F: \mathbb{R}^{n} \rightrightarrows \mathbb{R}^{n}$ is upper semicontinuous if for each $x \in \mathbb{R}^{n}$ and for all $\varepsilon>0$ there exists a $\delta>0$ such that for all $\xi \in B_{\delta}(x)$ we have $F(\xi) \subset F(x)+B_{\varepsilon}(0)$. It is Lipschitz continuous if there exists a constant $L>0$ and a neighborhood $\mathcal{O} \subset \mathbb{R}^{n}$ of $x \in \mathbb{R}^{n} \backslash\{0\}$ such that $F\left(x_{1}\right) \subset F\left(x_{2}\right)+B_{L\left|x_{1}-x_{2}\right|}(0)$ for all $x_{1}, x_{2} \in \mathcal{O}$, [3, Def. 1.4.5].

Assumption 2.1(i) ensures that solutions $\phi\left(\cdot ; x_{0}\right)$ : $[0, T) \rightarrow \mathbb{R}^{n},\left(T \in \mathbb{R}_{>0} \cup\{\infty\}\right)$ for an initial value $x_{0} \in \mathbb{R}^{n}$ satisfying the differential inclusion (1) for almost all $t \in[0, T)$ are absolutely continuous and in particular differentiable almost everywhere. The set of all solutions $\phi\left(\cdot ; x_{0}\right)$ with $\phi\left(0 ; x_{0}\right)=x_{0}$ is denoted by $S(x)$.

Solutions $\phi\left(\cdot ; x_{0}\right)$ are finite on a maximal time interval. To simplify the notation in the following, we define solutions $\phi\left(\cdot ; x_{0}\right): \mathbb{R} \rightarrow \mathbb{R}^{n} \cup\{ \pm \infty\}^{n}$ as extended real valued functions. In this case $\phi\left(\cdot ; x_{0}\right)$ is defined for all $t \in \mathbb{R}$ even in the case of finite escape time. Additionally, we will use the following convention:

- If $\phi_{i}\left(T ; x_{0}\right)= \pm \infty$ for $T>0$ and $i \in\{1, \ldots, n\}$, then $\phi_{i}\left(t ; x_{0}\right)= \pm \infty$ for all $t \geq T$.

- If $\phi_{i}\left(T ; x_{0}\right)= \pm \infty$ for $T<0$ and $i \in\{1, \ldots, n\}$, then $\phi_{i}\left(t ; x_{0}\right)= \pm \infty$ for all $t \leq T$.

For $t \in \mathbb{R}$ with $\left|\phi\left(t ; x_{0}\right)\right|=\infty$, the condition $\dot{\phi}\left(t ; x_{0}\right) \in$ $F\left(\phi\left(t ; x_{0}\right)\right)$ is satisfied by definition almost everywhere.

Instead of looking at a solution $\phi\left(t ; x_{0}\right)$ in forward time, $t \rightarrow \infty$, it will be useful in some sections to consider time reversal solutions, $t \rightarrow-\infty$, of the differential inclusion (1).
An extended real valued function $\psi\left(\cdot ; x_{0}\right): \mathbb{R} \rightarrow \mathbb{R}^{n} \cup$ $\{ \pm \infty\}^{n}$ is called a time reversal solution of the differential inclusion (1) if

$$
\psi\left(t ; x_{0}\right)=\phi\left(-t ; x_{0}\right)
$$

for a $\phi\left(\cdot ; x_{0}\right) \in S\left(x_{0}\right)$ for all $t \in \mathbb{R}$. A time reversal solution satisfies the differential inclusion

$$
\dot{x} \in-F(x), \quad x_{0} \in \mathbb{R}^{n},
$$

for almost all $t \in \mathbb{R}$.

Example 2.2 (Control system): As an example of a differential inclusion we consider the dynamical system

$$
\dot{x}=f(x, u)
$$

where $f: \mathbb{R}^{n} \times \mathbb{R}^{m} \rightarrow \mathbb{R}^{n}$ is Lipschitz continuous in the state $x \in \mathbb{R}^{n}$ and continuous in the input $u \in \mathbb{R}^{m}$. For a compact set $\mathcal{U} \in \mathbb{R}^{m}$ we define the differential inclusion

$$
F(x)=\overline{\operatorname{conv}}\left\{f(x, u) \in \mathbb{R}^{n} \mid u \in \mathcal{U}\right\} .
$$

Then the differential inclusion $\dot{x} \in F(x)$ satisfies Assumption 2.1, [12, Remark 4].

\section{B. The Dini derivative}

For a smooth function $\varphi: \mathbb{R}^{n} \rightarrow \mathbb{R}$ we denote the directional derivative at $x \in \mathbb{R}^{n}$ in direction $w \in \mathbb{R}^{n}$ by

$$
D \varphi(x ; w)=\langle\nabla \varphi(x), w\rangle
$$

Since solutions of the differential inclusion (1) are absolutely continuous but, in general, not necessarily continuously differentiable we use the Dini derivative to extend the notation of the directional derivative for Lipschitz continuous functions $\varphi$. For Lipschitz continuous functions $\varphi$ there are four definitions of the Dini derivative. The upper right, lower right, upper left, and the lower left Dini derivative at $x$ in direction $w \in \mathbb{R}^{n}$ are defined as:

$$
\begin{aligned}
& D^{+} \varphi(x ; w)=\limsup _{t \searrow 0} \frac{1}{t}(\varphi(x+t w)-\varphi(x)), \\
& D_{+} \varphi(x ; w)=\liminf _{t \searrow 0} \frac{1}{t}(\varphi(x+t w)-\varphi(x)), \\
& D^{-} \varphi(x ; w)=\limsup _{t \nearrow_{0}} \frac{1}{t}(\varphi(x+t w)-\varphi(x)), \\
& D_{-} \varphi(x ; w)=\liminf _{t{ }_{0}} \frac{1}{t}(\varphi(x+t w)-\varphi(x)) .
\end{aligned}
$$

For a Lipschitz continuous function $\varphi: \mathbb{R}^{n} \rightarrow \mathbb{R}$ the Dini derivatives are finite for all $x \in \mathbb{R}^{n}$ and $w \in \mathbb{R}^{n}$. If $\varphi$ is continuously differentiable in $x \in \mathbb{R}^{n}$, then all Dini derivatives coincide with the directional derivative, i.e.,

$$
\begin{aligned}
\langle\nabla \varphi(x), w\rangle & =D^{+} \varphi(x ; w)=D_{+} \varphi(x ; w) \\
& =D^{-} \varphi(x ; w)=D_{-} \varphi(x ; w) .
\end{aligned}
$$

However, note that the four definitions can indeed lead to different values for a Lipschitz continuous function.

Example 2.3: Let $\varphi:(-1,1) \rightarrow \mathbb{R}$ be defined as

$$
\varphi(x)=\left\{\begin{array}{cl}
x^{2} \sin \left(x^{-1}\right) & \text { for } x \in(-1,0], \\
2 x^{2} \sin \left(x^{-1}\right) & \text { for } x \in(0,1) .
\end{array}\right.
$$

For $x \in(-1,0)$ the derivative of $\varphi$ is given by

$$
\frac{d}{d x} \varphi(x)=2 x \sin \left(x^{-1}\right)-\cos \left(x^{-1}\right)
$$


which can be estimated by

$$
\sup _{x \in(-1,0)}\left|\frac{d}{d x} \varphi(x)\right| \leq \sup _{x \in(-1,0)}\left(\left|2 x \sin \left(x^{-1}\right)\right|+\left|\cos \left(x^{-1}\right)\right|\right) \leq 3 .
$$

In the same way we obtain $\sup _{x \in(0,1)}\left|\frac{d}{d x} \varphi(x)\right| \leq 6$ and,

$$
|\varphi(x)-\varphi(0)| \leq\left|2 x^{2} \sin \left(x^{-1}\right)\right| \leq 2\left|x^{2}\right| \leq 2|x-0|
$$

for all $x \in(-1,1)$. Thus, the function $\varphi$ is Lipschitz continuous with Lipschitz constant $L=6$. For the Dini derivatives at $x=0$ in direction $w=1$ we obtain

$$
\begin{array}{llrl}
D^{+} \varphi(0,1) & =2, & & D^{-} \varphi(0,1)=1, \\
D_{+} \varphi(0,1) & =-2, & & D_{-} \varphi(0,1)=-1 .
\end{array}
$$

The function $\varphi$ and the directional derivative for $x \in$ $[-0.2,0.2] \backslash\{0\}$ in the direction $w=1$ are visualized in Figure 1.
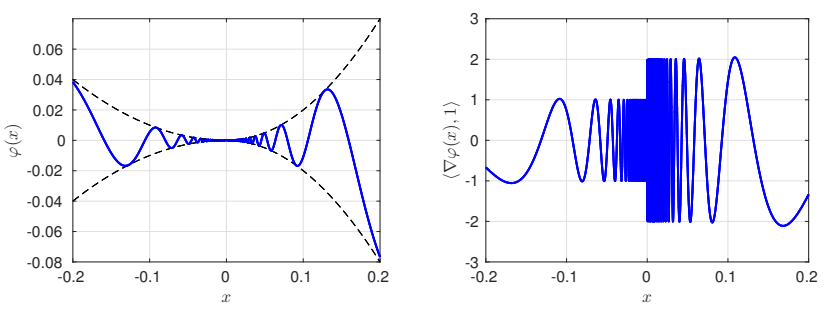

Fig. 1. The function $\varphi$ and its directional derivative on $[-0.2,0.2] \backslash\{0\}$.

For absolutely continuous solutions $\phi\left(\cdot ; x_{0}\right) \in S\left(x_{0}\right)$ of differential inclusion (1), at a fixed time $t$, the right Dini derivatives (4a)-(4b) indicate possible directions in forward time $t+\Delta t, \Delta t>0$, whereas the left Dini derivatives (4c)(4d) indicate possible directions in backward time $t-\Delta t$, $\Delta t>0$. Thus, for stability properties of the origin of the differential inclusion (1), the right Dini derivatives are used. Nevertheless the left Dini derivative will be important in Section V-A.

For a smooth function $\phi\left(\cdot ; x_{0}\right): \mathbb{R}_{\geq 0} \rightarrow \mathbb{R}^{n}$ and a smooth function $V: \mathbb{R}^{n} \rightarrow \mathbb{R}_{\geq 0}$ we use the notation

$$
\dot{V}\left(\phi\left(t ; x_{0}\right)\right)=\left\langle\nabla V\left(\phi\left(t ; x_{0}\right)\right), \dot{\phi}\left(t ; x_{0}\right)\right\rangle .
$$

to indicate the derivate of $V$ along the function $\phi$. If $\phi$ is absolutely continuous and $V$ is Lipschitz continuous, then (5) holds for almost all $t \in \mathbb{R}$.

\section{LYAPUNOV CHARACTERIZATIONS: KNOWN RESULTS AND MOTIVATING EXAMPLES}

Before we propose Lyapunov characterizations for differential inclusions for instability, we review results on LFs and CLFs for stability of differential inclusions and instability of ordinary differential equations (ODEs) in this section.

\section{A. Stability and instability of ordinary differential equations}

Stability properties of ODEs characterized through Lyapunov functions are well established. We consider ODEs

$$
\dot{x}=f(x), \quad x_{0} \in \mathbb{R}^{n},
$$

as a special form of the differential inclusion (1), with a Lipschitz continuous right-hand-side $f: \mathbb{R}^{n} \rightarrow \mathbb{R}^{n}$. In contrast to the generalized definition (1), solutions of (6) are unique and $S\left(x_{0}\right)$ contains only a single element for all $x_{0} \in \mathbb{R}^{n}$. Then, asymptotic stability of the origin can be characterized in the following ways.

Theorem 3.1: Consider the ODE (6). Then the following statements are equivalent.

(i) The origin $x=0$ is (uniformly) globally asymptotically stable.

(ii) There exists $\beta \in \mathcal{K} \mathcal{L}$ such that

$$
\left|\phi\left(t ; x_{0}\right)\right| \leq \beta\left(\left|x_{0}\right|, t\right) \quad \forall t \in \mathbb{R}_{\geq 0}, \quad \forall x_{0} \in \mathbb{R}^{n} .
$$

(iii) There exist a smooth function $V: \mathbb{R}^{n} \rightarrow \mathbb{R}, \alpha_{1}, \alpha_{2} \in$ $\mathcal{K}_{\infty}$, and $\rho \in \mathcal{P}$ such that

$$
\begin{gathered}
\alpha_{1}(|x|) \leq V(x) \leq \alpha_{2}(|x|) \\
\langle\nabla V(x), f(x)\rangle \leq-\rho(|x|)
\end{gathered}
$$

for all $x \in \mathbb{R}^{n}$.

The function $V$ in Theorem 3.1 is called an LF. For a proof of Theorem 3.1 we refer to [15, Prop. 2.5] for the equivalence between (i) and (ii) and [17, Thm. 6.2.3, Thm. 6.6.2] for the equivalence between (ii) and (iii).

Instability of an equilibrium is usually defined as not stable. However, there are different classifications of instability.

Definition 3.2 (Instability): Consider the ODE (6). The origin is

(i) unstable if for all $\varepsilon>0$ and for all $\delta>0$, there exists an $x_{0} \in B_{\delta}(0)$ and a $t \in \mathbb{R}_{\geq 0}$ with $\left|\phi\left(t ; x_{0}\right)\right|>\varepsilon$; and

(ii) completely unstable if for all $\varepsilon>0$ and for all $\delta>0$, for all $x_{0} \in B_{\delta}(0)$ there exists a $t_{x_{0}} \in \mathbb{R}_{\geq 0}$ such that $\left|\phi\left(t_{x_{0}} ; x_{0}\right)\right|>\varepsilon$.

Note that, as stated, the concepts in Definition 3.2 are essentially global as they are stated for all $\varepsilon>0$; that is, trajectories eventually leave every neighborhood of the origin. Local versions are easily obtained by restricting $\varepsilon$. For instability of the origin, a similar result to Theorem 3.1 can be stated.

Theorem 3.3 ([17, Lyapunov's instability Thm. 6.2.8]): Consider the ODE (6). Assume there exists a smooth function $C: \mathbb{R}^{n} \rightarrow \mathbb{R}$ and $\rho \in \mathcal{P}$ such that

$$
\langle\nabla C(x), f(x)\rangle \geq \rho(|x|), \quad \forall x \in \mathbb{R}^{n} .
$$

(i) If for all $\varepsilon>0$ there exists $x \in B_{\varepsilon}(0)$ such that $C(x)>0$, then the equilibrium is unstable.

(ii) If $C(x)>0$ for all $x \in \mathbb{R}^{n} \backslash\{0\}$, then the equilibrium is completely unstable.

There are more general results on instability for ODEs where the most general goes back to Chetaev, [6]. However, for the following discussion, Theorem 3.3 is sufficient. To distinguish between stability and instability results we refer to $V$ as a $\mathrm{LF}$ and $C$ as a Chetaev function (CF) in the following.

Example 3.4: To illustrate complete instability, instability, and asymptotic stability we consider three linear differential 
equations and their solutions:

$$
\begin{aligned}
f_{1}(x)=\left(\begin{array}{c}
x_{1} \\
x_{2}
\end{array}\right), & \phi_{1}\left(t ; x_{0}\right)=\left(\begin{array}{l}
x_{1,0} e^{t} \\
x_{2,0} e^{t}
\end{array}\right), \\
f_{2}(x)=\left(\begin{array}{r}
-x_{1} \\
x_{2}
\end{array}\right), & \phi_{2}\left(t ; x_{0}\right)=\left(\begin{array}{l}
x_{1,0} e^{-t} \\
x_{2,0} e^{t}
\end{array}\right), \\
f_{3}(x)=\left(\begin{array}{r}
-x_{1} \\
-x_{2}
\end{array}\right), & \phi_{3}\left(t ; x_{0}\right)=\left(\begin{array}{l}
x_{1,0} e^{-t} \\
x_{2,0} e^{-t}
\end{array}\right) .
\end{aligned}
$$

For the ODE (8a) all solutions $\phi_{1}\left(\cdot ; x_{0}\right), x_{0} \neq 0$ satisfy $\left|\phi_{1}\left(t ; x_{0}\right)\right| \rightarrow \infty$ for $t \rightarrow \infty$, which indicates that the origin is completely unstable. Similarly, for $(8 \mathrm{c})$ the solutions satisfy $\left|\phi_{3}\left(t ; x_{0}\right)\right| \rightarrow 0$ for $t \rightarrow \infty$ for all $x_{0} \in \mathbb{R}^{n}$, showing asymptotic stability of the equilibrium.

For (8b) there exist initial values $x_{0}=(0 c)^{T}, c \neq 0$, such that $\left|\phi_{2}\left(t ; x_{0}\right)\right| \rightarrow \infty$ for $t \rightarrow \infty$ as well $x_{0}=(c 0)^{T}$, $c \neq 0$, such that $\left|\phi_{2}\left(t ; x_{0}\right)\right| \rightarrow 0$ for $t \rightarrow \infty$, i.e., the origin $x=0$ is unstable but not completely unstable.

These properties can also be rigorously verified using the LF $V_{1}(x)=x^{T} x$ and the CFs $C_{2}(x)=-x_{1}^{2}+x_{2}^{2}$ and $C_{3}(x)=x^{T} x$.

If instability is considered in the context of destabilization and obstacle avoidance, it is clear that instability is not the appropriate concept since it does not guarantee that all solutions drift away from the origin. Thus, we will concentrate on complete instability in the remainder of this paper. A definition of complete instability in terms of comparison functions as well as the extension to differential inclusions has not been introduced in the literature yet (to the best of our knowledge).

\section{B. (Control) LFs: Stability of Differential inclusions}

Since solutions of differential inclusions are not unique in general, we consider two different definitions describing asymptotic stability, or equivalently $\mathcal{K} \mathcal{L}$-stability.

Definition 3.5: The differential inclusion (1) is strongly $\mathcal{K} \mathcal{L}$-stable with respect to the equilibrium $0 \in \mathbb{R}^{n}$ if there exists $\beta \in \mathcal{K} \mathcal{L}$ such that, for all $x_{0} \in \mathbb{R}^{n}$ every solution $\phi \in \mathcal{S}\left(x_{0}\right)$ satisfies

$$
\left|\phi\left(t ; x_{0}\right)\right| \leq \beta\left(\left|x_{0}\right|, t\right), \quad \forall t \in \mathbb{R}_{\geq 0} .
$$

Definition 3.6: The differential inclusion (1) is weakly $\mathcal{K} \mathcal{L}$-stable with respect to the equilibrium $0 \in \mathbb{R}^{n}$ if there exists $\beta \in \mathcal{K} \mathcal{L}$ such that, for all $x_{0} \in \mathbb{R}^{n}$ there exists $\phi \in \mathcal{S}\left(x_{0}\right)$ so that

$$
\left|\phi\left(t ; x_{0}\right)\right| \leq \beta\left(\left|x_{0}\right|, t\right), \quad \forall t \in \mathbb{R}_{\geq 0} .
$$

Strong $\mathcal{K} \mathcal{L}$-stability describes robustness properties of the equilibrium, whereas weak $\mathcal{K} \mathcal{L}$-stability indicates that a system is stabilizable. Corresponding to strong and weak stability, LFs and CLFs a can be defined.

Definition 3.7 (Robust Lyapunov function): A Lipschitz continuous function $V: \mathbb{R}^{n} \rightarrow \mathbb{R}$ is called a LF for the differential inclusion (1) if there exist $\alpha_{1}, \alpha_{2} \in \mathcal{K}_{\infty}$ and $\rho \in \mathcal{P}$ such that

$$
\begin{array}{r}
\alpha_{1}(|x|) \leq V(x) \leq \alpha_{2}(|x|) \\
\max _{w \in F(x)} D^{+} V(x ; w) \leq-\rho(|x|)
\end{array}
$$

holds for all $x \in \mathbb{R}^{n}$.

Definition 3.8 (Control Lyapunov function): A Lipschitz continuous function $V: \mathbb{R}^{n} \rightarrow \mathbb{R}$ is called a CLF for the differential inclusion (1) if there exist $\alpha_{1}, \alpha_{2} \in \mathcal{K}_{\infty}$ and $\rho \in \mathcal{P}$ such that

$$
\begin{array}{r}
\alpha_{1}(|x|) \leq V(x) \leq \alpha_{2}(|x|) \\
\min _{w \in F(x)} D_{+} V(x ; w) \leq-\rho(|x|)
\end{array}
$$

holds for all $x \in \mathbb{R}^{n}$.

With these definitions, the following connections between stability and the existence of (control) LFs have been derived.

Theorem 3.9 ([7, Thm. 1.1],[8]): Suppose F satisfies Assumption 2.1. Then the following are equivalent.

(i) The differential inclusion (1) is strongly $\mathcal{K} \mathcal{L}$-stable according to Definition 3.5.

(ii) There exists a smooth LF according to Definition 3.7.

Thus, for strong $\mathcal{K} \mathcal{L}$-stability it is even possible to assume that the robust Lyapunov function is smooth. In the case of weak $\mathcal{K} \mathcal{L}$-stability this is not the case.

Theorem 3.10: Suppose $F$ satisfies Assumption 2.1. Then the following are equivalent.

(i) The differential inclusion (1) is weakly $\mathcal{K} \mathcal{L}$-stable according to Definition 3.6.

(ii) There exists a CLF according to Definition 3.8.

Theorem 3.10 based upon results from [20], [18] and [12]. Theorem 3.9 and Theorem 3.10 extend the classical stability result for ODEs. Since in the case of ODEs with Lipschitz continuous right-hand-side, $\mathcal{S}\left(x_{0}\right)$ contains only a single element the definitions of strong and weak $\mathcal{K} \mathcal{L}$-stability coincide and are equivalent to uniform global asymptotic stability [15, Prop. 2.5].

\section{INSTABILITY CHARACTERIZATIONS FOR DIFFERENTIAL INCLUSIONS}

In this section we will mirror existing stability results to describe complete instability properties of differential inclusions. To this end, we will use definitions similar to Definitions 3.5 and 3.6 by using appropriate comparison functions and we will extend Theorem 3.3 by generalizing the concept of Chetaev functions.

\section{A. Complete instability of differential inclusions}

In the stability context, $\mathcal{K} \mathcal{L}$-functions provide an upper bound for solutions of differential inclusions. To establish instability, by contrast, a lower bound for the solutions is needed.

Definition $4.1\left(\mathcal{K}_{\infty} \mathcal{K}\right.$ - and $\mathcal{K}_{\infty} \mathcal{K}_{\infty}$-functions $):$ Consider the continuous function $\kappa: \mathbb{R}_{\geq 0}^{2} \rightarrow \mathbb{R}_{\geq 0}$.

(i) The function $\kappa$ is said to be of class $\mathcal{K}_{\infty} \mathcal{K}\left(\kappa \in \mathcal{K}_{\infty} \mathcal{K}\right)$ if $\kappa(\cdot, s) \in \mathcal{K}_{\infty}$ for all $s \in \mathbb{R}_{\geq 0}$ and $\kappa(s, \cdot)-\kappa(s, 0) \in$ $\mathcal{K}$ for all $s \in \mathbb{R}_{>0}$.

(ii) The function $\kappa$ is said to be of class $\mathcal{K}_{\infty} \mathcal{K}_{\infty}$ ( $\kappa \in$ $\left.\mathcal{K}_{\infty} \mathcal{K}_{\infty}\right)$ if $\kappa(\cdot, s) \in \mathcal{K}_{\infty}$ for all $s \in \mathbb{R}_{\geq 0}$ and $\kappa(s, \cdot)-$ $\kappa(s, 0) \in \mathcal{K}_{\infty}$ for all $s \in \mathbb{R}_{>0}$. 
Example 4.2: As an example consider the function $h$ : $\mathbb{R}_{\geq 0}^{2} \rightarrow \mathbb{R}_{\geq 0}, h(s, t)=C e^{\lambda t} s$, and $C>0$. It holds that $h(\cdot, t) \in \mathcal{K}_{\infty}^{-}$for all $t \in \mathbb{R}_{\geq 0}$. Additionally, $h(s, \cdot)-h(s, 0) \in$ $\mathcal{K}_{\infty}$ for all $s>0$ and $\lambda>0$, and $h(s, \cdot) \in \mathcal{L}$ for all $s \geq 0$ and $\lambda<0$. Thus $h \in \mathcal{K}_{\infty} \mathcal{K}_{\infty}$ for $\lambda>0$ and $h \in \mathcal{K} \mathcal{L}$ for $\lambda<0$.

With these definitions, we can mirror Definitions 3.5 and 3.6 to characterize complete instability of differential inclusions.

Definition 4.3: The equilibrium $0 \in \mathbb{R}^{n}$ is strongly completely unstable with respect to the differential inclusion (1) if there exists $\kappa \in \mathcal{K}_{\infty} \mathcal{K}_{\infty}$ such that, for all $x_{0} \in \mathbb{R}^{n}$ every solution $\phi \in \mathcal{S}\left(x_{0}\right)$ satisfies

$$
\left|\phi\left(t ; x_{0}\right)\right| \geq \kappa\left(\left|x_{0}\right|, t\right), \quad \forall t \in \mathbb{R}_{\geq 0} .
$$

Definition 4.4: The equilibrium $0 \in \mathbb{R}^{n}$ is weakly completely unstable with respect to the differential inclusion (1) if there exists $\kappa \in \mathcal{K}_{\infty} \mathcal{K}_{\infty}$ such that, for all $x_{0} \in \mathbb{R}^{n}$ there exists $\phi \in \mathcal{S}\left(x_{0}\right)$ so that

$$
\left|\phi\left(t ; x_{0}\right)\right| \geq \kappa\left(\left|x_{0}\right|, t\right), \quad \forall t \in \mathbb{R}_{\geq 0} .
$$

Remark 4.5: Note that in the literature the term $\mathcal{K} \mathcal{L}$ stability has been established, despite the fact that, for uniform global asymptotic stability, $\beta(\cdot, t)$ generally needs to be of class $\mathcal{K}_{\infty}$ (not only class $\mathcal{K}$ ) for all $t \geq 0$.

The following example illustrates why $\kappa \in \mathcal{K}_{\infty} \mathcal{K}_{\infty}$ is chosen to characterize instability rather than $\kappa \in \mathcal{K}_{\infty} \mathcal{K}$.

Example 4.6: Consider the ODE $\dot{x}=0$ which trivially has the origin as a stable equilibrium point. Assume that $\kappa \in$ $\mathcal{K}_{\infty} \mathcal{K}$ is used in Definition 4.3 to define complete instability and consider the function

$$
\kappa(r, t)=\frac{1}{2} r\left(2-e^{-t}\right) \in \mathcal{K}_{\infty} \mathcal{K} .
$$

For all $x_{0} \in \mathbb{R}^{n}$ and for all $t \in \mathbb{R}_{\geq 0}$ it holds that

$$
\left|\phi\left(t ; x_{0}\right)\right|=\left|x_{0}\right| \geq \kappa\left(\left|x_{0}\right|\right)=\frac{1}{2}\left|x_{0}\right|\left(2-e^{-t}\right) \geq \frac{1}{2}\left|x_{0}\right|
$$

which would imply that the origin is completely unstable. Since the origin of the ODE is stable but not asymptically stable (i.e., $\mathcal{K} \mathcal{L}$-stable) $\mathcal{K}_{\infty} \mathcal{K}$-functions are not the right conceptual tool to describe equivalences between $\mathcal{K} \mathcal{L}$-stability and complete instability for systems in forward time and the corresponding time reversed system.

The $\mathcal{K}_{\infty} \mathcal{K}_{\infty}$-function ensures that a solution satisfies $\left|\phi\left(t ; x_{0}\right)\right| \rightarrow \infty$ for $t \rightarrow \infty$ for all $x_{0} \in \mathbb{R}^{n} \backslash\{0\}$. If this is not desirable, in obstacle avoidance, for example, where only a certain neighborhood around the origin is supposed to be left, the following local definition can be used instead.

Definition 4.7: Let $\mathcal{O} \subset \mathbb{R}^{n}$ be an open neighborhood containing the origin $0 \in \mathcal{O}$. The equilibrium $0 \in \mathbb{R}^{n}$ is locally strongly completely unstable with respect to the differential inclusion (1) and the neighborhood $\mathcal{O}$ if there exists a $\kappa \in \mathcal{K}_{\infty} \mathcal{K}_{\infty}$ such that, for all $x_{0} \in \mathcal{O}$ every solution $\phi \in \mathcal{S}\left(x_{0}\right)$ satisfies

$$
\left|\phi\left(t ; x_{0}\right)\right| \geq \kappa\left(\left|x_{0}\right|, t\right)
$$

for all $t \in \mathbb{R}_{>0}$ such that $\phi\left(t ; x_{0}\right) \in \mathcal{O}$.

Local weak complete instability can be defined in the same way.

\section{B. Sufficient conditions for complete instability}

In this section we will derive sufficient conditions for complete instability of differential inclusions in terms of Chetaev functions.

Definition 4.8 (Robust Chetaev function): A Lipschitz continuous function $C: \mathbb{R}^{n} \rightarrow \mathbb{R}$ is called a $\mathrm{CF}$ for the differential inclusion (1) if there exist $\alpha_{1}, \alpha_{2} \in \mathcal{K}_{\infty}$ and $\rho \in \mathcal{P}$ such that

$$
\begin{aligned}
\alpha_{1}(|x|) \leq C(x) & \leq \alpha_{2}(|x|) \\
\min _{w \in F(x)} D_{+} C(x ; w) & \geq \rho(|x|)
\end{aligned}
$$

holds for all $x \in \mathbb{R}^{n}$.

Definition 4.9 (Control Chetaev function): A Lipschitz continuous function $C: \mathbb{R}^{n} \rightarrow \mathbb{R}$ is called a control $\mathrm{CF}$ (CCF) for the differential inclusion (1) if there exist $\alpha_{1}, \alpha_{2} \in \mathcal{K}_{\infty}$ and $\rho \in \mathcal{P}$ such that

$$
\begin{aligned}
\alpha_{1}(|x|) \leq C(x) & \leq \alpha_{2}(|x|) \\
\max _{w \in F(x)} D^{+} C(x ; w) & \geq \rho(|x|)
\end{aligned}
$$

holds for all $x \in \mathbb{R}^{n}$.

To be able to show that the existence of a $(\mathrm{C}) \mathrm{CF}$ implies complete instability we need the following comparison principle.

Lemma 4.10: For any function $\rho \in \mathcal{P}$ there exists a $\kappa \in$ $\mathcal{K}_{\infty} \mathcal{K}_{\infty}$ such that if $y:[0, T] \rightarrow \mathbb{R},\left(T \in \mathbb{R}_{>0} \cup\{\infty\}\right)$ is a locally absolutely continuous function which satisfies the differential inequality

$$
\dot{y}(t) \geq \lambda \rho(y(t))
$$

for almost all $t \in[0, T]$, for some $\lambda>0$ with $y(0)=y_{0} \in$ $\mathbb{R}_{\geq 0}$ then

$$
y(t) \geq \kappa\left(y_{0}, \lambda t\right), \quad \forall t \in[0, T] .
$$

A similar result providing an upper bound in terms of a $\mathcal{K} \mathcal{L}$ estimate is well known and can for example be found in [21, Lemma A.4] or [10, Lemma 20]. The proof of Lemma 4.10 is given in the Appendix.

Theorem 4.11: Suppose $F$ satisfies Assumption 2.1. Assume there exists a $\mathrm{CF}$ according to Definition 4.8. Then the differential inclusion (1) is strongly completely unstable according to Definition 4.3.

Proof: We follow the lines of [8] (who themselves refer to [14]). We define the set-valued map $H: \mathbb{R}_{\geq 0} \rightrightarrows \mathbb{R}^{n}$,

$$
H(v)=\left\{x \in \mathbb{R}^{n} \mid C(x)=v\right\}
$$

and the function $\gamma: \mathbb{R}_{\geq 0} \rightarrow \mathbb{R}_{\geq 0}$,

$$
\gamma(v)=\min \{\rho(|x|) \mid x \in H(v)\} .
$$

(Lipschitz) continuity of $C$ and continuity of $\rho$ imply continuity of $\gamma$. Moreover, it holds that $\gamma \in \mathcal{P}$ since $\rho \in \mathcal{P}$ and $C(x)>0$ for all $x \neq 0$.

Since $C$ is locally Lipschitz, $C(\phi(\cdot ; x))$ is absolutely continuous. Hence, due to the definition of the function $\gamma$ 
and due to condition (19) an arbitrary solution $\phi(\cdot ; x) \in S(x)$ satisfies

$$
\begin{aligned}
\frac{d}{d t} C(\phi(t ; x)) & =\langle\nabla C(\phi(t ; x)), \dot{\phi}(t ; x)\rangle \\
& \geq \rho(|\phi(t ; x)|) \geq \gamma(C(\phi(t ; x)))
\end{aligned}
$$

for almost all $t \in \mathbb{R}_{\geq 0}$. With these definitions, Lemma 4.10 can be applied to $\frac{d}{d t} C(\phi(t ; x)) \geq \gamma(C(\phi(t ; x))$, for all $x \in$ $\mathbb{R}^{n}$, which yields a function $\kappa \in \mathcal{K}_{\infty} \mathcal{K}_{\infty}$ such that

$$
C(\phi(t ; x)) \geq \kappa(C(x), t)
$$

for all $\phi(\cdot ; x) \in S(x)$ and for all $t \in[0, T]$. The inequalities (18) lead to the estimate

$$
\left.\alpha_{2}(|\phi(t ; x)|) \geq C(\phi(t ; x)) \geq \kappa(C(x), t)\right) \geq \kappa\left(\alpha_{1}(|x|), t\right),
$$

i.e., $|\phi(t ; x)| \geq \alpha_{2}^{-1} \circ \kappa\left(\alpha_{1}(|x|), t\right)$. Thus, the assertion follows with the function $\alpha_{2}^{-1} \circ \kappa\left(\alpha_{1}(\cdot), \cdot\right) \in \mathcal{K}_{\infty} \mathcal{K}_{\infty}$.

Theorem 4.12: Suppose $F$ satisfies Assumption 2.1. Assume there exists a CCF according to Definition 4.9. Then the differential inclusion (1) is weakly completely unstable according to Definition 4.4.

Proof: The proof is similar to the proof of Theorem 4.11. Let $H$ and $\gamma$ be defined in (24) and (25), respectively. Assume there exists $\phi(\cdot ; x) \in S(x)$ such that

$$
\begin{aligned}
\frac{d}{d t} C(\phi(t ; x)) & =\langle\nabla C(\phi(t ; x)), \dot{\phi}(t ; x)\rangle \\
& \geq \frac{1}{4} \rho(|\phi(t ; x)|) \geq \frac{1}{4} \gamma(C(\phi(t ; x))
\end{aligned}
$$

for almost all $t \in \mathbb{R}_{\geq 0}$.

Again, Lemma 4.10 applied to (27) provides a function $\kappa \in \mathcal{K} \mathcal{K}_{\infty}$ such that $C(\phi(t ; x)) \geq \kappa(C(x), t)$ and

$$
|\phi(t ; x)| \geq \alpha_{2}^{-1} \circ \kappa\left(\alpha_{1}(|x|), t\right)=\tilde{\kappa}(|x|, t)
$$

with $\tilde{\kappa} \in \mathcal{K}_{\infty} \mathcal{K}_{\infty}$.

The proof is complete if we can show that the pointwise condition (21) ensures that for all $x \in \mathbb{R}^{n}$ there exists $\phi(\cdot ; x) \in \mathcal{S}(x)$ satisfying property (27) for almost all $t \in$ $\mathbb{R}_{\geq 0}$.

We assume to the contrary, that there exists an $x \in \mathbb{R}^{n}$ and an $\Gamma>0$ such that all solutions $\phi(\cdot ; x) \in S(x)$ satisfy

$$
\frac{d}{d t} C(\phi(t ; x))<\frac{1}{4} \rho(|\phi(t ; x)|)
$$

for all $t$ in a set of non-zero measure contained in $[0, \Gamma]$.

We choose an $\varepsilon>0$ such that $\frac{1}{2} \rho(|y|)<\rho(|x|)$ for all $y \in B_{\varepsilon}(x)$. Due to condition (21), there exists a $\tilde{w} \in F(x)$ such that

$$
D^{+} C(x ; \tilde{w}) \geq \rho(|x|) .
$$

Since $F$ is Lipschitz continuous there exists a Lipschitz continuous function $w:[0, \Gamma] \rightarrow \mathbb{R}^{n}$ such that $\phi(\cdot ; x) \in$ $S(x), \dot{\phi}(t ; x)=w(t)$ for all $t \in[0, \Gamma]$ and $w(0)=\tilde{w}$ (and $w(t) \in F(\phi(t ; x))$. Note that $\phi(\cdot ; x)$ is Lipschitz continuous. From assumption (28) and $\frac{1}{2} \rho(|\phi(t ; x)|)<\rho(|x|)$, we obtain the condition

$$
\frac{1}{t}\left(C(\phi(t ; x))-C(\phi(0 ; x))<\frac{1}{2} \rho(|x|)\right.
$$

for all $t \in(0, \Gamma)$ such that $\phi(t ; x) \in B_{\varepsilon}(x)$. Since the left-hand-side is Lipschitz continuous, we can take the limit superior for $t \rightarrow 0$ on both sides, which contradicts (29) and thus the assumption (28) was wrong.

To sum up, this implies that for all $x \in \mathbb{R}^{n}$ there exists a $\phi(\cdot ; x) \in S(x)$ such that the increase condition (27) is satisfied for all $t \in[0, \Gamma]$ where $\Gamma>0$. Since this argument can be applied iteratively to the initial value $\phi(\Gamma ; x)$, there exists a solution $\phi(\cdot ; x) \in S(x)$ such that (27) is satisfied for all $t \geq 0$.

Theorem 3.9 and 3.10, as well as the results of [13], [22], indicate that the converses of Theorems 4.11 and 4.12 should also hold, i.e., that complete instability implies the existence of a (control) CF. These results are left for future research. Instead, we turn to the connection between LFs and CFs as well as stability in forward time and instability in backward time of dynamical systems in the sequel.

\section{CONNECTIONS BETWEEN STABILITY AND INSTABILITY PROPERTIES}

In this section we investigate connections between the existence of (control) LFs and (control) CFs. In the same way, we investigate connections between stability properties of equilibria for dynamical systems in forward time and instability properties in backward time.

\section{A. (Control) LFs versus (Control) CFs}

Theorem 5.1: Let $V: \mathbb{R}^{n} \rightarrow \mathbb{R}$ be a smooth function. Suppose $F$ satisfies Assumption 2.1. Then $V$ is a (control) LF for system (1) if and only if $V$ is a (control) CF for system (2).

Proof: Let $V$ be a smooth CLF of system (1). Then estimate (14) can be written as

$$
-\max _{w \in F(x)}-\langle\nabla V(x), w\rangle \leq-\rho(|x|) .
$$

Thus, the inequality

$$
\max _{w \in F(x)}\langle\nabla V(x),-w\rangle=\max _{w \in-F(x)}\langle\nabla V(x), w\rangle \geq \rho(|x|)
$$

holds, which shows that $V$ is a CCF for the time reversal system (2). The relation between smooth LFs and smooth CFs can be shown in the same way.

A similar result for nonsmooth (control) LFs and (control) CFs does not hold. To see this, assume that $V$ is a CLF for the differential inclusion (1), i.e., the condition

$$
-\rho(|x|) \geq \min _{w \in F(x)} D_{+} V(x ; w)
$$

holds for all $x \in \mathbb{R}^{n}$. Using the definition of the lower right Dini derivative, this condition can be equivalently written as

$$
\begin{aligned}
\rho(|x|) & \leq \max _{w \in F(x)}-D_{+} V(x ; w) \\
& =\max _{w \in F(x)}-\liminf _{t \searrow 0} \frac{1}{t}(V(x+t w)-V(x)) \\
& =\max _{w \in F(x)} \limsup _{t \searrow 0}-\frac{1}{t}(V(x+t w)-V(x)) \\
& =\max _{w \in F(x)} \limsup _{t \nearrow 0} \frac{1}{t}(V(x-t w)-V(x)) \\
& =\max _{w \in-F(x)} \limsup _{t \nearrow 0} \frac{1}{t}(V(x+t w)-V(x)) \\
& =\max _{w \in-F(x)} D^{-} V(x ; w) .
\end{aligned}
$$


The calculations above show that the right Dini derivative becomes a left Dini derivative, which cannot be used to compute an increasing direction for the time reversal system (2). The same arguments hold if we start with a nonsmooth LF instead of a nonsmooth CLF.

The fact that the existence of a nonsmooth CLF for $\dot{x} \in$ $F(x)$ indeed does not imply that there exists a nonsmooth CCF for $\dot{x} \in-F(x)$ can be observed on the example of Artstein's circles [2].

Example 5.2 (Artstein's circles): The dynamical system $\dot{x}=f(x, u)$ described by

$$
\begin{aligned}
& \dot{x}_{1}(t)=\left(-x_{1}(t)^{2}+x_{2}(t)^{2}\right) u(t) \\
& \dot{x}_{2}(t)=\left(-2 x_{1}(t) x_{2}(t)\right) u(t)
\end{aligned}
$$

is known as Artstein's circles in the literature. For $u \in$ $[-1,1]=\mathcal{U}$ and $F(x)=\overline{\operatorname{conv}}\{f(x, u) \mid u \in \mathcal{U}\}$ the dynamics can be described in the form of a differential inclusion (1). The function

$$
V(x)=\sqrt{4 x_{1}^{2}+3 x_{2}^{2}}-\left|x_{1}\right|
$$

is a CLF in the Dini sense according to Definition 3.8, which implies weak $\mathcal{K} \mathcal{L}$-stability according to Theorem 3.10 . Nevertheless, the time reversal system is not weakly completely unstable since all solutions of Artstein's circles with initial value $x \in \mathbb{R}^{2} \backslash(\mathbb{R} \times\{0\})$ are bounded for all $t \in \mathbb{R}_{\geq 0}$.

More explicitly all solutions of the dynamical system (30) are described through circles, where the radius of the circle is defined by the initial value. The input $u$ can only change the direction (left or right) and the velocity of the solution. For any potential CCF $C$ there needs to exists at least one point $\tilde{x} \in \mathbb{R}^{n}$ on a circle corresponding to any initial value $x_{0}$ where no increasing direction $D^{+} C(\tilde{x} ; w)>0$ exists. This is true for initial values arbitrarily close to the origin.

We summarize the observation in the following corollary.

Corollary 5.3: Let $F$ satisfy Assumption 2.1. Weak $\mathcal{K} \mathcal{L}$ stability of the origin of $\dot{x} \in F(x)$ is not equivalent to weak complete instability of the origin of $\dot{x} \in-F(x)$.

This result shows that even though there are similarities between stability in forward time and instability in backward time, instability results cannot simply be defined by mirroring known results from stability theory. In particular in the destabilization of dynamical systems there are several open questions left for future research.

\section{B. Stability versus Instability}

The example of Artstein's circles shows that weak $\mathcal{K} \mathcal{L}$ stability in forward time is not equivalent to weak complete instability in backward time.

By contrast, we conjecture that strong $\mathcal{K} \mathcal{L}$-stability in forward time is equivalent to strong complete instability in backward time. Indeed, strong $\mathcal{K} \mathcal{L}$-stability in forward time is equivalent to the existence of a smooth strong Lyapunov function (Theorem 3.9), which according to Section V-A is a strong Chetaev function for the system in backward time. Theorem 4.11 then implies that $\dot{x} \in-F(x)$ is strongly completely unstable. The converse statement, i.e., strong complete instability of $\dot{x} \in-F(x)$ implies strong $\mathcal{K} \mathcal{L}$-stability, holds if one can show that the converse of Theorem 4.11 is true.

Conjecture 5.4: Let $F$ satisfy Assumption 2.1. Strong $\mathcal{K} \mathcal{L}$-stability of the origin of $\dot{x} \in F(x)$ is equivalent to strong complete instability of the origin of $\dot{x} \in-F(x)$.

If a linear system is strongly $\mathcal{K} \mathcal{L}$-stable, a $\mathcal{K}_{\infty} \mathcal{K}_{\infty}$ function showing strong complete instability of the time reversal system can be easily derived. However, a similar relation for nonlinear systems does not hold, which we illustrate in the following two examples.

Example 5.5 (Linear dynamical systems): Consider the linear system $\dot{x}=A x$ and let $\sigma_{\mathbb{R}}(A)$ be the set of the real parts of the eigenvalues of $A$. Assume that the linear system is asymptotically stable, i.e., $a_{\max }=\max _{a \in \sigma_{\mathbb{R}}(A)} a<0$. Then there exists a $\underline{C} \in \mathbb{R}_{>0}$ such that

$$
\left|\phi\left(t ; x_{0}\right)\right|=\left|e^{A t} x_{0}\right| \leq \underline{C} e^{a_{\max } t}\left|x_{0}\right|=\beta\left(\left|x_{0}\right|, t\right) .
$$

for all $x_{0} \in \mathbb{R}^{n}$. Similarly, for the time reversal system there exists a $\bar{C} \in \mathbb{R}_{>0}$ such that

$$
\left|\psi\left(t ; x_{0}\right)\right|=\left|e^{-A t} x_{0}\right| \geq \bar{C} e^{-a_{\max } t}\left|x_{0}\right|=\kappa\left(\left|x_{0}\right|, t\right) .
$$

for all $x_{0} \in \mathbb{R}^{n}$

Example 5.6: Consider the nonlinear system $\dot{x}=-x^{3}$ and its timed reversed counterpart $\dot{x}=x^{3}$ with the solutions

$$
\phi(t ; x)=\frac{x}{\sqrt{1+2 t x^{2}}} \quad \text { and } \quad \psi(t ; x)=\frac{x}{\sqrt{1-2 t x^{2}}},
$$

respectively. The system in forward time is (weakly and strongly) $\mathcal{K} \mathcal{L}$-stable with $\mathcal{K} \mathcal{L}$-function

$$
\beta(r, t)=\frac{r}{\sqrt{1+2 t r^{2}}}+e^{-t} r .
$$

The time reversal system has finite escape time. Thus, the solution cannot be used as a $\mathcal{K}_{\infty} \mathcal{K}_{\infty}$-estimate. However, it is easy to show that

$$
\left|\psi\left(t ; x_{0}\right)\right| \geq \kappa\left(\left|x_{0}\right|, t\right)=\left|x_{0}\right|(t+1)
$$

\section{CONCLUSions}

In this paper we introduced CFs and CCFs as an analogue to LFs and CLFs to describe complete instability properties of differential inclusions. In this context we derived sufficient conditions for complete instability and we derived connections between the existence of (control) LFs for dynamical systems in forward time and the existence of (control) CFs for the corresponding time reversal system. Future work includes deriving necessary conditions for (control) CFs, mirroring known converse Lyapunov theorems.

\section{APPENDIX}

The following proof is similar to the proof of the comparison principle provided in [10, Lemma 20].

Proof of Lemma 4.10.

We first demonstrate the case $\lambda=1$. Let the assumptions of the lemma be satisfied for $\lambda=1$. We define the function $\hat{\rho}(s)=\min \{s, \rho(s)\}$. Observe that

$$
\dot{y}(t) \geq \lambda \rho(y(t)) \geq \lambda \hat{\rho}(y(t))
$$


holds for all $t \in[0, T]$. For $s \in(0, \infty)$ we define the function

$$
\eta(s)=\int_{1}^{s} \frac{1}{\hat{\rho}(\tau)} d \tau .
$$

We observe that $\eta(s)$ is continuously differentiable and strictly increasing for $s \in(0, \infty)$. Due to the condition $s \geq \hat{\rho}(s)$ for all $s \in(0,1)$ it holds that

$$
\int_{s}^{1} \frac{1}{\tau} d \tau \leq \int_{s}^{1} \frac{1}{\hat{\rho}(\tau)} d \tau=-\eta(s)
$$

which implies $\lim _{s \searrow 0} \eta(s)=-\infty$. For $s \in[1, \infty)$ it holds that

$$
\int_{1}^{s} \frac{1}{\tau} d \tau \leq \int_{1}^{s} \frac{1}{\hat{\rho}(\tau)} d \tau=\eta(s)
$$

which implies $\lim _{s \rightarrow \infty} \eta(s)=\infty$.

Thus, $\eta:(0, \infty) \rightarrow(-\infty, \infty)$ and, since $\eta$ is continuous and strictly increasing, $\eta^{-1}:(-\infty, \infty) \rightarrow(0, \infty)$ is also continuous and strictly increasing. We define the function

$$
\kappa(s, t)= \begin{cases}0, & s=0 \\ \eta^{-1}(\eta(s)+t), & s>0\end{cases}
$$

for all $s, t \in \mathbb{R}_{\geq 0}$. Since $\eta^{-1}(\cdot)$ is unbounded it holds that $\eta^{-1}(\eta(s)+\cdot)-\eta^{-1}(\eta(s)) \in \mathcal{K}_{\infty}$ for all $s>0$. For all $s>0$, $t \geq 0$ it holds that

$$
0 \leq \eta^{-1}(\eta(s)+t) \geq \eta^{-1}(\eta(s))=s,
$$

which implies that $\kappa$ is continuous (in particular at $(s, t)=$ $(0,0))$ and $\kappa(\cdot, t) \in \mathcal{K}_{\infty}$ for all $t \geq 0$ and we can conclude that $\kappa \in \mathcal{K}_{\infty} \mathcal{K}_{\infty}$. If $y_{0}=0$, any function $\kappa \in \mathcal{K}_{\infty} \mathcal{K}_{\infty}$ satisfies $y(t) \geq 0=\kappa(0, \lambda t)$ for all $t \in[0, T]$. In the case $y_{0}>0$, condition (22) ensures that $y(t) \geq y_{0}$, and thus $\hat{\rho}(y(t)) \neq 0$, for all $t \in[0, T]$. This implies that for all $y_{0}>0$ inequality (22) can be rewritten as

$$
\frac{\dot{y}(t)}{\hat{\rho}(y(t))} \geq 1
$$

and integration over both sides leads to

$$
\int_{y_{0}}^{y(t)} \frac{1}{\hat{\rho}(r)} d r=\int_{0}^{t} \frac{\dot{y}(\tau)}{\hat{\rho}(y(\tau))} d \tau \geq t .
$$

Using the definition of the function $\eta$ we obtain $\eta(y(t))-$ $\eta\left(y_{0}\right) \geq t$ or equivalently

$$
y(t) \geq \eta^{-1}\left(\eta\left(y_{0}\right)+t\right)
$$

which shows the assertion for $\lambda=1$.

By taking the time rescaling $\tau=\lambda t$, we see that $\dot{y}(t) \geq$ $\lambda \hat{\rho}(y(t))$ for almost all $t \in[0, T]$ becomes

$$
\frac{d}{d \tau} y(\tau / \lambda) \geq \hat{\rho}(y(\tau / \lambda))
$$

for almost all $\tau \in[0, \lambda T]$. Following the steps above, we have a function $\kappa \in \mathcal{K}_{\infty} \mathcal{K}_{\infty}$ such that $y(\tau / \lambda) \geq \kappa\left(y_{0}, \tau\right)$ for all $\tau \in[0, \lambda T]$ and hence $y(t) \geq \kappa\left(y_{0}, \lambda t\right)$, for all $t \in[0, T]$.

\section{REFERENCES}

[1] A. D. Ames, X. Xu, J. W. Grizzle, and P. Tabuada. Control barrier function based quadratic programs for safety critical systems. IEEE Transactions on Automatic Control, 62(8):3861-3876, 2017.

[2] Z. Artstein. Stabilization with relaxed controls. Nonlinear Analysis, 7(11):1163-1173, 1983.

[3] J.-P. Aubin and H. Frankowska. Set-Valued Analysis. Springer Science \& Business Media, 2009.

[4] P. Braun and C. M. Kellett. On (the existence of) control Lyapunov barrier functions. pages 1-19, 2018. Submitted, Preprint: https://epub.uni-bayreuth.de/3522/1/CLBFs_submission_pbraun.pdf.

[5] P. Braun., C. M. Kellett, and L. Zaccarian. Unsafe point avoidance in linear state feedback. pages $1-8,2018$. Submitted, Preprint: https://epub.uni-bayreuth.de/3584/1/pbraun_submission.pdf.

[6] N. G. Chetaev. The Stability of Motion. Pergamon Press, 1962. Translated from the 2nd Edition in Russian of 1956.

[7] F. Clarke. Lyapunov functions and discontinuous stabilizing feedback. Annual Reviews in Control, 35(1):13-33, 2011.

[8] F. H. Clarke, Y. S. Ledyaev, and R. J. Stern. Asymptotic stability and smooth Lyapunov functions. Journal of Differential Equations, 149(1):69-114, 1998.

[9] D. Efimov, W. Perruquetti, and M. Petreczky. On necessary conditions of instability and design of destabilizing controls. In Proceedings of the 53rd IEEE Conference on Decision and Control, pages 3915-3917, Los Angeles, CA, USA, 2014.

[10] C. M. Kellett. A compendium of comparsion function results. Mathematics of Controls, Signals and Systems, 26(3):339-374, 2014.

[11] C. M. Kellett. Classical converse theorems in Lyapunov's second method. Discrete and Continuous Dynamical Systems, Series B, 20(8):2333-2360, 2015.

[12] C. M. Kellett and A. R. Teel. Weak converse Lyapunov theorems and control-Lyapunov functions. SIAM J. Control and Optimization, 42(6):1934-1959, 2004.

[13] N. N. Krasovskii. Transformation of the theorem of A. M. Lyapunov's second method and questions of first-order stability of motion. Prikl. Mat. Mekh., 20(2):255-265, 1956. [Russian].

[14] V. Lakshmikantham and S. Leela. Differential and Integral Inequalities: Theory and Applications. Academic Press, 1969.

[15] Y. Lin, E. D. Sontag, and Y. Wang. A smooth converse Lyapunov theorem for robust stability. SIAM J. Control and Optimization, 34(1):124-160, 1996

[16] A. M. Lyapunov. The general problem of the stability of motion. International Journal of Control, 55(3):531-534, 1992. (Original in Russian, Math. Soc. of Kharkov, 1892).

[17] A. Michel, L. Hou, and D. Liu. Stability of Dynamical Systems. Springer, 2008.

[18] L. Rifford. Existence of Lipschitz and semiconcave control-Lyapunov functions. SIAM Journal on Control and Optimization, 39(4):10431064,2000

[19] R. G. Sanfelice and C. Prieur. Robust supervisory control for uniting two output-feedback hybrid controllers with different objectives. Automatica, 49(7):1958-1969, 2013.

[20] E. D. Sontag. A Lyapunov-like characterization of asymptotic controllability. SIAM J. Control and Optimization, 21:462-471, 1983.

[21] E. D. Sontag and Y. Wang. Lyapunov characterizations of input to output stability. SIAM J. Control and Optimization, 39(1):226-249, 2000.

[22] I. Vrkoč. A general theorem of Chetaev. Chechoslovak Mathematics Journal, 5(4):451-461, 1955. [Russian].

[23] P. Wieland and F. Allgöwer. Constructive safety using control barrier functions. IFAC Proceedings Volumes, 40(12):462-467, 2007. 